\title{
Impending Rupture With a Twisted Huge Appendiceal Mucocele Treated With Totally Laparoscopic Resection: A Case Series
}

\author{
Hong-min $\mathrm{Ahn}^{1,2}$, Si-Hak Lee ${ }^{1,2,3}$ \\ ${ }^{1}$ Department of Surgery and ${ }^{2}$ Research Institute for Convergence of Biomedical Science and Technology, Pusan National University Yangsan \\ Hospital, Yangsan; ${ }^{3}$ Department of Surgery, Pusan National University School of Medicine, Yangsan, Korea
}

Torsion of the appendix is rare, and appendiceal mucocele can be one of its causes. The first case was of a 49-year-old man who visited the emergency room (ER) for abdominal pain. Abdominal computed tomography (CT) showed appendiceal mucocele with suspected torsion and rupture. The patient underwent laparoscopic exploration and appendectomy. The second case was of a 69-year-old man who visited the ER for epigastric pain. Abdominal CT showed suspicious appendiceal mucocele with ischemic change, indicating torsion of the appendix. The twisted appendix was successfully removed by laparoscopic exploration. An appendiceal mucocele is one of the causes of twisted appendix. With torsion, the mucocele can be diagnosed as rupture by ischemia which may lead to pseudomyxoma peritonei. For this reason, open laparotomy has traditionally been preferred. However, an unruptured appendiceal mucocele or impending rupture with torsion of the appendiceal mucocele can be treated with totally laparoscopic surgery.

\section{Keywords: Torsion of appendix; Appendiceal mucocele; Laparoscopic appendectomy}

\section{INTRODUCTION}

Torsion of the appendix is a rare condition and can be divided into 2 categories; primary and secondary torsion. Primary torsion of the appendix is defined as the event of ischemic or necrotic change with dilation of the appendix without any primary lesion. The shape of the appendix is considered one of the factors for primary torsion, such as a long appendix or fan-shaped mesoappendix with a narrow base $[1,2]$. Meanwhile, the secondary torsion of the appendix involves abnormality in the appendix. A variety of lesions that cause appendiceal torsion, such as fecalith, cystadenoma, or appendiceal mucocele, have been reported [3].

Received: Mar 10, 2020 - Revised: May 7, 2020 - Accepted: May 14, 2020 Correspondence to: Si-Hak Lee, M.D.

Department of Surgery, Pusan National University Yangsan Hospital, 20 Geumo-ro, Mulgeum-eup, Yangsan 50612, Korea

Tel: +82-55-360-2124, Fax: +82-55-360-2154

E-mail: ghost109@hanmail.net

ORCID: https://orcid.org/0000-0002-1923-4777

(c) 2021 The Korean Society of Coloproctology

This is an open-access article distributed under the terms of the Creative Commons Attribution NonCommercial License (https://creativecommons.org/licenses/by-nc/4.0) which permits unrestricted noncommercial use, distribution, and reproduction in any medium, provided the original work is properly cited.
Appendiceal mucocele is a rare lesion in the appendix. Without a twisting event, it is usually asymptomatic and often diagnosed incidentally. Perforation of an appendiceal mucocele may lead to catastrophic results, such as pseudomyxoma peritonei (PMP), depending on the histological finding of mucocele $[4,5]$. This is why it is greatly important to prevent rupture of the mucocele during surgery. In our cases, we successfully removed the huge twisted appendiceal mucoceles without iatrogenic rupture by totally laparoscopic surgery.

\section{CASE REPORTS}

\section{Case 1}

A 49-year-old man with no previous medical history visited the emergency room (ER) with diffused, intermittent abdominal pain. His symptoms started a day before the visit, and no other gastrointestinal symptoms, such as nausea, vomiting, constipation, and diarrhea, were noted. One tender point was found in the right lower quadrant of the abdomen, which was slightly below the McBurney point. It was negative for peritoneal irritation sign.

The vital signs on arrival were stable, with a blood pressure of $120 / 80 \mathrm{mmHg}$, heart rate of 66 beats per minute, and body tem- 
perature of $36.2^{\circ} \mathrm{C}$. Initially, laboratory and simple X-ray studies were performed, and none of the results showed any abnormality; leukocyte count was $8,310 / \mu \mathrm{L}$ with $70.1 \%$ of segment neutrophils, and C-reactive protein (CRP) level was $0.04 \mathrm{mg} / \mathrm{dL}$ (normal range, 0 to $0.5 \mathrm{mg} / \mathrm{dL}$ ). For further pain evaluation, abdominal computed tomography (CT) was performed by one of the radiologists in our facility. The result showed $3.4-\mathrm{cm}$ dilatation of the appendix, which indicated appendiceal mucocele (Fig. 1). The possibility of torsion and suspicious wall defects with increased periappendiceal fat strands was also found through CT; therefore, we decided to perform emergency laparoscopic exploration for suspicion of both twisted and ruptured appendiceal mucocele.

The surgery was started with 3 small incisions for trocars: 1 trocar of 11-mm on the subumbilical incision and 2 trocars of 5-mm
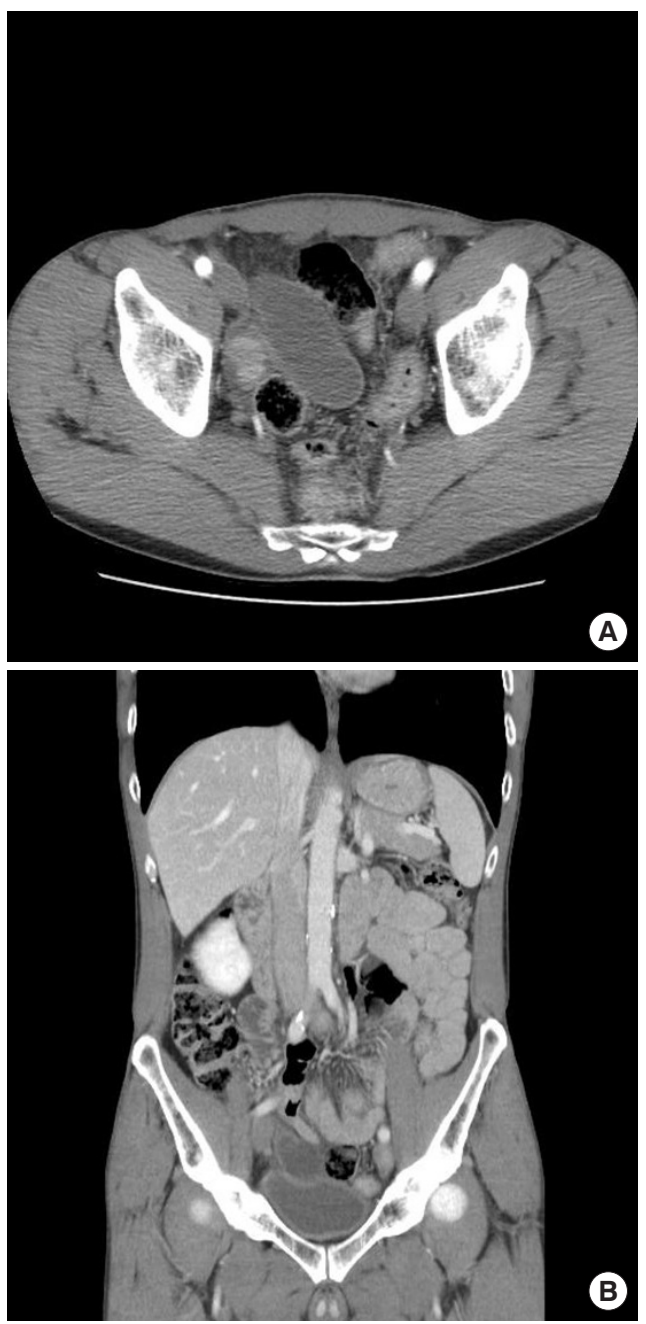

Fig. 1. Abdominal computed tomography for case 1. (A) Axial view. A 3.4-cm dilatation of the appendix with suspicious torsion and rupture of the appendiceal mucocele. (B) Coronal view. Suspicious wall defects in the appendix. on both suprapubic and left lower quadrant incisions. By surgical exploration, counterclockwise torsion of huge appendiceal mucocele was confirmed (Fig. 2). Releasing the base of the twisted appendix gently, we found that the mucocele had not ruptured. Eventually, a simple laparoscopic appendectomy was performed. By gently grasping the mucocele, appendectomy was performed without any iatrogenic injury of the appendiceal mucocele. The specimen was safely removed using an endobag without rupturing (Fig. 3). Based on the pathologic findings, the confirmative diagnosis was low-grade appendiceal mucinous neoplasm (LAMN) with dissection through the muscularis propria and serosa, and the size of the specimen was recorded as length of $9 \mathrm{~cm}$ and width of $3.5 \mathrm{~cm}$. The patient was discharged without any complications and transferred to the colorectal surgical department for regular checkup.

\section{Case 2}

A 69-year-old man with a history of totally laparoscopic distal gastrectomy for advanced gastric cancer visited the ER for epigastric pain. His symptoms, with mild nausea, started 9 hours before the visit. The physical examination showed right upper and lower abdominal quadrant tenderness. It was negative for peritoneal irritation sign.

The vital signs on arrival were stable, with a blood pressure of $137 / 76 \mathrm{mmHg}$, heart rate of 72 beats per minute, and body temperature of $36.0^{\circ} \mathrm{C}$. In the initial laboratory study, leukocytosis $(15,420 / \mu \mathrm{L})$ with elevated neutrophil segment $(83.4 \%)$ and normal CRP level $(0.04 \mathrm{mg} / \mathrm{dL})$ was noted. Abdominal CT was performed and showed both suspicious appendiceal mucocele and appendicitis. We also suspected ischemic rupture with torsion of

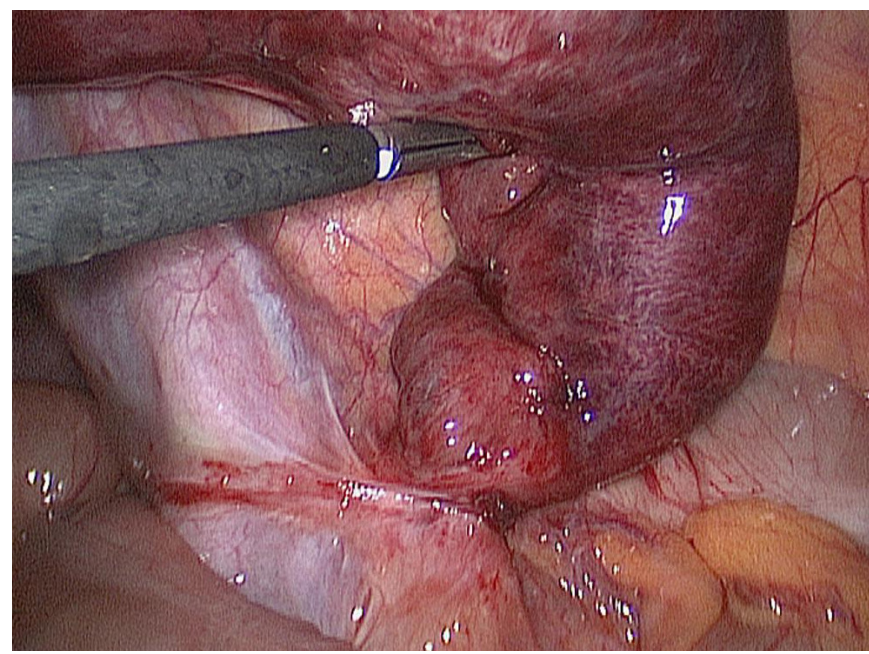

Fig. 2. Laparoscopic exploration of case 1. Impending rupture with a twisted appendix was found. 


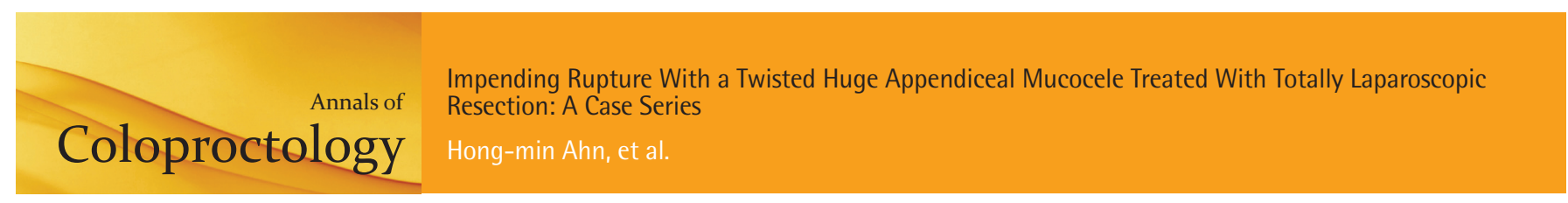

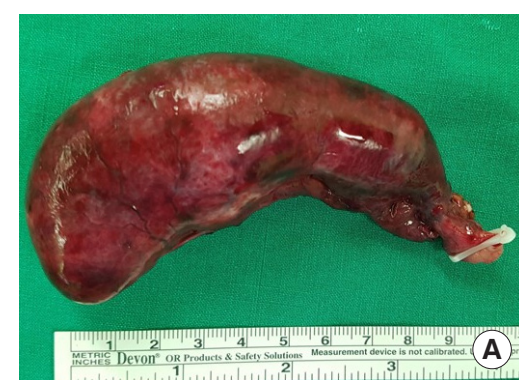

Fig. 3. Specimen of an appendix in case 1 . Gross length of approximately $10 \mathrm{~cm}$ (A) and width of approximately 5 to $6 \mathrm{~cm}(\mathrm{~B})$.
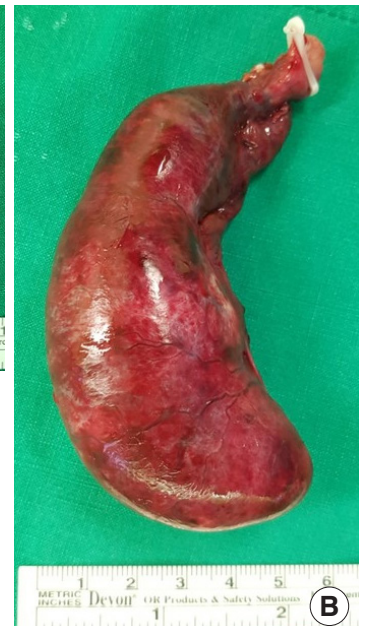

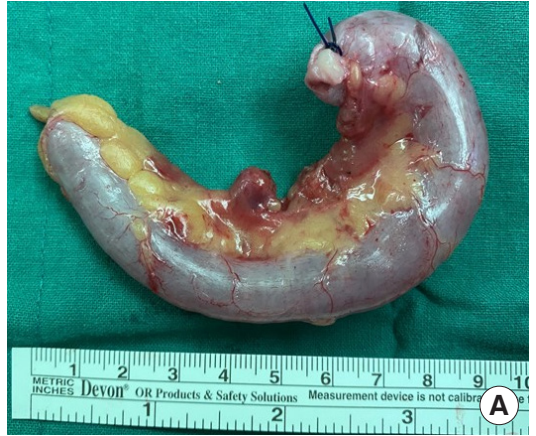

Fig. 5. Specimen of an appendix in case 2 . Gross length of approximately $11 \mathrm{~cm}$ in J shape (A) and width of approximately 3 to $4 \mathrm{~cm}(\mathrm{~B})$.

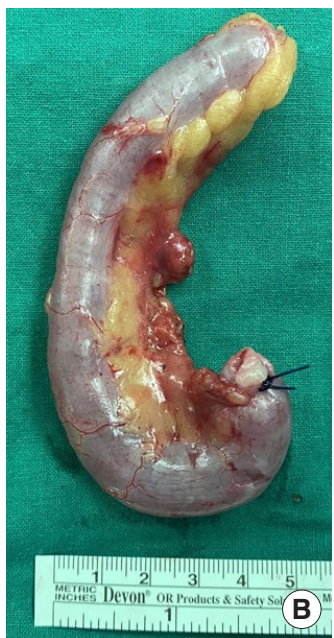

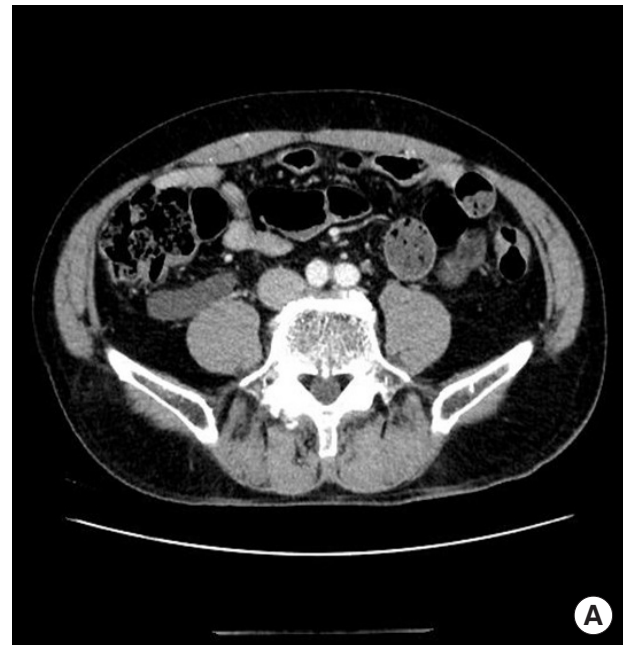

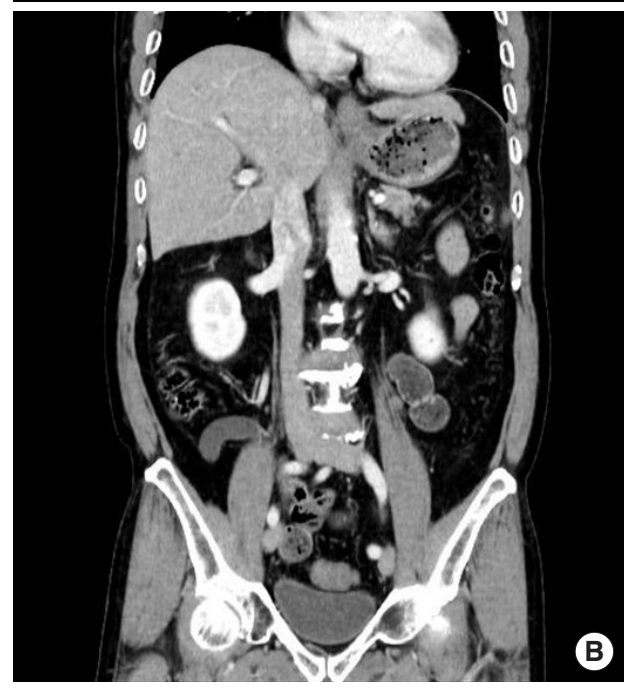

Fig. 4. Abdominal computed tomography for case 2. (A) Axial view. A decrease in the enhancement of a long appendix. (B) Coronal view. Suspicious torsion of the appendix. the appendix since the enhancement of the appendiceal wall on CT was decreased (Fig. 4). Laparoscopic exploration was selected as the confirmation and treatment modality.

Through the exploration, a long and thick appendix was twisted counterclockwise without rupture of the appendiceal wall. By ligation of the appendiceal artery with release of the twisted appendix, we found that the base of the appendix is intact for simple removal. Thus, laparoscopic appendectomy was performed using the endobag to safely remove the resected specimen (Fig. 5). Final histologic findings confirmed the diagnosis of appendiceal mucocele, and its size was recorded as length of $11 \mathrm{~cm}$ and width of 1.6 $\mathrm{cm}$. The patient was discharged without any complications.

This study was reviewed and approved by the ethics committee at our institution (No. 05-2021-107).

\section{DISCUSSION}

Appendiceal mucocele is a rare condition in the appendix. The incidence of this disease is approximately $0.1 \%$ to $0.7 \%$ of appendiceal specimens, including incidental findings $[4,6]$. The complications of appendiceal mucocele include inflammation, invagination, obstruction, bleeding, fistula formation, rupture, and torsion [7]. Torsion of the appendix was first described in the British literature in 1918 by Payne $[3,8]$. Since then, only a few cases have been reported, and most studies on appendiceal torsion have been based on case reports [3]. For this reason, the definite mechanism and causes of twisting the appendix still remain unknown. By the literature reviews, the age at presenting the torsion of appendix was similar to that of acute appendicitis, which occurs throughout all ages on both males and females [3]. The morphology of the appendix such as long appendix, fan-shaped mesoappendix, or appendix with narrow base may increase the risk of primary torsion $[1,2]$. However, some studies assume that secondary torsion of the appendix, like our presented cases, could be similar to ovarian torsion, which twists around its own axis, with a fulcrum of 
the short segment of the normal appendix between the cystic mass and cecum $[9,10]$.

Histologically, appendiceal mucocele can be categorized into 4 causes: retention cyst, mucosal hyperplasia, LAMN or mucinous cystadenoma, and mucinous cystadenocarcinoma [4-6, 9]. More than half of mucinous tumors (52\% to $58 \%$ ) are considered malignant tumors of the appendix $[5,6]$. Spontaneous or iatrogenic rupture of appendiceal mucocele may lead to dissemination of epithelial cells that produce mucins into the peritoneal cavity. This may cause PMP, which may affect the long-term prognosis of the disease, especially with malignant mucocele [4-7]. Moreover, this may lead to life-long complicated management, including debulking, peritonectomy, and heated intraperitoneal chemotherapy (HIPEC) $[4,6]$. Even with these great efforts, according to previous studies, the 5-year survival rate of PMP is only $25 \%$ [6]. Because of this, it is extremely important to prevent iatrogenic rupture of mucocele during surgical removal of the appendix [11, 12]. To prevent rupture, open appendectomy has been preferred and is even considered as the standard plan for appendiceal mucocele removal [4-6]. However, with advancement of the technique and equipment of laparoscopic surgery, minimally invasive surgery, including laparoscopic appendectomy, has become one of the modalities of appendiceal mucocele resection $[13,14]$.

Different strategies have been recommended in the management of appendiceal mucocele in previous case reports, and the choice of surgery varied from simple appendectomy to right hemicolectomy [4, 11]. Dhage-Ivatury and Sugarbaker [11] suggested that laparotomy for a thorough exploration would be necessary with the surgical treatment of appendiceal mucocele, since the risk of rupturing the mucocele by laparoscopic procedure is high. Furthermore, they recommended HIPEC for perforated mucocele and secondary surgery for the positive margin at resection. Cecectomy has been suggested for negative appendiceal lymph nodes, and right hemicolectomy for positive lymph nodes [11]. However, Morano et al. [4] suggested laparoscopic surgery as a safe and effective technique for appendiceal mucocele removal. Focusing on the pathology, further treatment plans have been suggested as patients with resection margin-positive LAMN were recommended colonoscopy for surveillance and those with mucinous cystadenocarcinoma were recommended right hemicolectomy [4].

In both our cases, we anticipated minor rupture with twisted appendiceal mucocele based on the CT results, and open laparotomy could have been the treatment of choice, according to the previous standards $[4,11]$. However, we initially decided on the laparoscopic approach for exploration and found secondary torsion of the appendix due to appendiceal mucocele without rupture. Laparoscopic appendectomy was performed in both cases, and the initial surgery was discontinued, anticipating the possibility of a second-look surgery if the pathology shows malignancy or a positive result on the resection margin. With both of these experiences, it is safe to suggest laparoscopic exploration as an initial plan for torsion of appendiceal mucocele with impending rupture. With confirmation of the nonperforated appendix, the primary treatment can be followed with laparoscopic appendectomy.

From our 2 cases, we have accomplished 2 messages on torsion of appendiceal mucocele. One is that, even though abdominal CT has shown ruptured appendiceal mucocele, laparoscopic exploration to confirm the ruptured state should be recommended instead of opening the abdomen immediately. The other message is that, even with a twisted huge appendiceal mucocele, if it is not ruptured, laparoscopic surgery could be one of the initial treatment choices to be considered. Therefore, in case of a huge appendiceal mucocele with torsion, laparoscopic exploration should be a necessary step to prevent excessive procedures.

\section{CONFLICT OF INTEREST}

No potential conflict of interest relevant to this article was reported.

\section{ACKNOWLEDGMENTS}

This study was supported by a 2020 research grant from Pusan National University Yangsan Hospital.

\section{REFERENCES}

1. Kitagawa M, Kotani T, Yamano T, Tsurudome H, Hatakeyama T, Kuriu Y, et al. Secondary torsion of vermiform appendix with mucinous cystadenoma. Case Rep Gastroenterol 2007;1:32-7.

2. Lee CH, Lee MR, Kim JC, Kang MJ, Jeong YJ. Torsion of a mucocele of the vermiform appendix: a case report and review of the literature. J Korean Surg Soc 2011;81(Suppl 1):S47-50.

3. Darbyshire D, Sutton P, Kosai N, Varghese J. Torsion of the vermiform appendix: a review of the literature. West Lond Med J 2011;3:14-8.

4. Morano WF, Gleeson EM, Sullivan SH, Padmanaban V, Mapow $\mathrm{BL}$, Shewokis PA, et al. Clinicopathological features and management of appendiceal mucoceles: a systematic review. Am Surg 2018;84:273-81.

5. B B SK, Jasuja P. Appendiceal mucocele: a rare case report. Int J Surg Case Rep 2019;58:21-5.

6. Motsumi MJ, Motlaleselelo P, Ayane G, Sesay SO, Valdes JR. A case report of a giant appendiceal mucocele and literature review. Pan Afr Med J 2017;28:106.

7. Stark C, Jousi M, Enholm B. Preoperative assessment and treatment of appendiceal mucocele complicated by acute torsion: a case report. BMC Res Notes 2014;7:1.

8. Carter AE. Torsion of the appendix. Postgrad Med J 1959;35:6712.

9. Aggarwal N, Bhargava A. Appendiceal mucocele secondary to torsion in an asymptomatic patient. J Surg Case Rep 2019;2019: rjz241. 


\section{Coloproctology Hong-min Ahn, et al.}

10. Rudloff U, Malhotra S. Volvulus of an appendiceal mucocele: report of a case. Surg Today 2007;37:514-7.

11. Dhage-Ivatury S, Sugarbaker PH. Update on the surgical approach to mucocele of the appendix. J Am Coll Surg 2006;202:680-4.

12. Lorenzon L, De Dominicis C, Virgilio E, Balducci G. The appropriate management of an appendiceal mucocele. BMJ Case Rep 2015;2015:bcr2014209045.
13. Ju YT, Park ST, Ha WS, Hong SC, Lee YJ, Jung EJ, et al. Laparoscopic resection of a appendiceal mucocele. J Korean Surg Soc 2011; 80(Suppl 1):S21-5.

14. Park KJ, Choi HJ, Kim SH. Laparoscopic approach to mucocele of appendiceal mucinous cystadenoma: feasibility and short-term outcomes in 24 consecutive cases. Surg Endosc 2015;29:3179-83. 